\title{
Mitarbeitende binden, Mitarbeitende finden
}

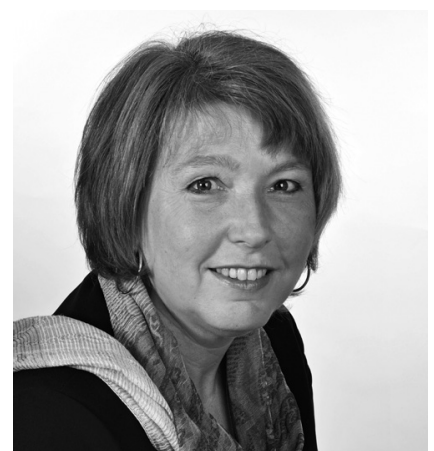

VON MARTINA POLLERT Martina Pollert studierte Wirtschaftswissenschaften an der Universität des Saarlandes. Von 1994 bis 2006 war sie im Diakonischen Werk der Evangelischen Kirche im Rheinland als Referentin für die Organisationsund Wirtschaftsberatung der Diakonie- und Sozialstationen der Rheinischen Kirche sowie für die Verhandlungsführung mit den Kranken- und Pflegekassen zuständig. Berufsbegleitend absolvierte sie eine Ausbildung zur Organisationsberaterin. Seit 2007 ist sie Geschäftsführerin der Diakoniestationen Essen gGmbH. m.pollert@diakoniestationenessen.de

\author{
Insbesondere in der Pflege ist es schwierig, \\ Mitarbeiterinnen und Mitarbeiter dauerhaft an das \\ Unternehmen zu binden und neue Fachkräfte zu finden. \\ Ein Konzept für Personalmarketing kann dabei hilfreich \\ sein, wie das Beispiel der Diakoniestationen Essen zeigt.
}

Die Leistungsfähigkeit sozialwirtschaftlicher Unternehmen hängt ganz entscheidend davon ab, wie es in Zukunft gelingt, die Mitarbeiterzufriedenheit zu erhöhen. Deutlich wird dies in der von Organisationsberatern viel zitierten Frage: "Jeden Abend verlässt 80 Prozent des Kapitals Ihr Unternehmen. Was tun Sie, damit es morgen zurückkehrt?"

Darüber hinaus ist es seit etwa sechs Jahren immer schwieriger geworden, geeignetes Personal zu gewinnen. Hierfür verantwortlich ist einerseits der demographische Wandel, andererseits das häufig negativ behaftete Image des Pflegeberufs vor allem bei jungen Menschen.

Dieser Umstand machte sich bei der Diakoniestationen Essen g GmbH vor allem ab Mitte des Jahres 2008 und insbesondere im Jahr 2009 bemerkbar. Kontinuierliches Wachstum der vergangenen Jahre führte zu Personalengpässen, die durch Mehrarbeitsstunden und mit Hilfe von Mitarbeitenden von Zeitarbeitsfirmen kompensiert werden mussten. Einerseits führten die notwendigen Mehrarbeitsstunden zu erheblichen Belastungen bei unseren Mitarbeitenden, andererseits war der Einsatz von Zeitarbeitskräften bis zu 35 Prozent teurer als das eigene Personal.

Vor diesem Hintergrund setzte sich bei den Diakoniestationen Essen die Erkenntnis durch, dass zufriedene Mitarbeitende nicht nur den »zufriedenen Kunden « nach sich ziehen, sondern auch unser Image als Arbeitgeber stärken und die Chance auf die Gewinnung neuer Mitarbeitenden erhöht. Der »Ressource Mitarbeitender« kommt damit zunehmend eine wirtschaftliche Schlüsselrolle zu.

\section{Strategie der kleinen Schritte}

Gemeinsam mit unseren Führungskräften und unserer Mitarbeitervertretung verständigten wir uns auf ein mitarbeiterorientiertes Personalmarketingkonzept. Dies bedeutet, dass unserer Personalpolitik an den Bedürfnissen der Mitarbeitenden orientiert wird, mit dem Ziel, gegenwärtige Mitarbeitende zu halten, zu motivieren und neue Mitarbeitende zu gewinnen. Dieses Konzept ist nicht statisch, sondern wird kontinuierlich weiterentwickelt.

Aufgrund unserer begrenzten personellen und finanziellen Ressourcen, erfolgte die Umsetzung schrittweise. In den Jahren 2007 bis 2009 beschäftigten wir uns zunächst mit dem Thema Personalbindung, ab 2009 mit dem Thema Personalgewinnung. In einer Analyse wurde die Ist-Situation erfasst. Auf dieser Grundlage haben wir jeweils die nächsten Schritte miteinander vereinbart.

\section{Erster Meilenstein für die Personalbindung: Commitment}

Die Personalbindung hatte für uns zunächst die höchste Priorität. Dabei ging es nicht um juristische Sachverhalte, sondern vielmehr um ein "psychologisches Band « zwischen unseren Mitarbeitenden und uns als Unternehmen. Dieses psychologische Band, die Mitarbeiterbindung, verstehen wir als die Verbundenheit, $\mathrm{Zu}$ gehörigkeit und Identifikation, die Mitarbeiter gegenüber uns als Unternehmen empfinden und erleben. Im Vergleich zur Arbeitszufriedenheit zeichnet sich Com- 
mitment eher durch Langfristigkeit und Stabilität aus. Arbeitszufriedenheit kann durch kurzfristig hohe Krankenquoten gestört sein, steigt aber nach Rückgang des Krankenstandes wieder an.

Um das Commitment weiter auszubauen, bietet das Mitarbeitergespräch eine praktische Unterstützung zur Präzisierung und Reflexion der Verbundenheit unserer Mitarbeiter. Einen Leitfaden für die Durchführung von strukturierten Mitarbeiterjahresgesprächen haben wir im Jahr 2007 mit unseren Führungskräften und der Mitarbeitervertretung entwickelt. Dieser Leitfaden liegt jedem Mitarbeitenden vor dem Gespräch vor, so dass sich sowohl die Leitung als auch die Mitarbeitenden auf diese Gespräche vorbereiten können. Einerseits wurden im Rahmen dieser Gespräche die Zusammenarbeit zwischen den Führungskräften und den Mitarbeitenden auf allen Hierarchieebenen reflektiert, andererseits wurden individuelle Fortund Weiterbildungswünsche der Mitarbeitenden erfasst und entsprechende Qualifizierungen ermöglicht.

Weitere Elemente zur Steigerung des Commitment waren: Einführung von Mitarbeiterfesten alle zwei Jahre; regelmäßige Teilnahme am Essener Firmenlauf; persönliche Geburtstagsbriefe der Geschäftsführung für alle Mitarbeitenden; Einführung eines Betriebliches Wiedereingliederungsmanagement; Verstärkung des Arbeitsschutzes und schrittweise Entwicklung eines Gesundheitsmanagements.

\section{Zweiter Meilenstein für die Personalbindung: Mitarbeiterbefragung}

Ein nächster großer Meilenstein war eine strukturierte Mitarbeiterbefragung durch ein externes Institut. Sie diente als sinnvolles Mittel, um Maßnahmen zur Mitarbeiterbindung nicht ins Blaue hinein zu konzipieren, sondern an den realen Bedürfnissen der Mitarbeiter auszurichten. Hierbei traten einige überraschende Ergebnisse zu Tage:

- Nicht alle Mitarbeitenden waren über ihre tariflichen und übertariflichen Ansprüche durchgängig informiert.

- Elementare Unternehmensabläufe waren nicht bekannt.

- Nicht alle angeboten Leistungen der Diakoniestationen waren bekannt.
- Die Führungskultur war in den einzelnen Standorten sehr unterschiedlich.

- Die Zufriedenheit der Mitarbeitenden in den einzelnen Standorten war sehr unterschiedlich.

- Die individuelle Empfindung der Arbeitsbelastung war sehr unterschiedlich.

Die Ergebnisse der Befragung wurden mit den Führungskräften, aber auch mit den Mitarbeitenden diskutiert. Hieraus wurden Prioritäten in der Weiterarbeit entwickelt. Zunächst stellten wir uns dem Thema Arbeitszufriedenheit. In einem zweiten Schritt folgten Überlegungen zu dem Thema Unternehmenskommunikation.

\section{Dritter Meilenstein für die Personalbindung: Arbeitszufriedenheit}

Arbeitszufriedenheit steht in einer direkten Beziehung zu Fehlzeiten und Kündigungsverhalten und ist somit für die Mitarbeiterbindung von zentraler Bedeutung. Um Mitarbeiter dauerhaft zum Verbleib bei uns zu bewegen, gibt es verschiedene Motivatoren. Neben dem Motivationsfaktor, der sich aus der Tätigkeit mit den Patienten selbst ergibt, kommt ein anerkennender Führungsstil hinzu. Weitere Motivatoren erhalten un-
Einen etwas längeren Zeitraum nahmen die Überarbeitung unseres Leitbildes und die Verständigung auf gemeinsame Führungsleitlinien in Anspruch (vgl. Abb. S. 14). Allen Mitarbeitenden liegen die Arbeitsergebnisse vor. Neue Mitarbeitende erhalten beides bei Einstellung ausgehändigt.

Durch die Gewährung eines zusätzlich freien Tages zu Unterbrechung des 12-Tage-Arbeitsrhythmus versuchten wir die Arbeitsbelastung der Mitarbeitenden zu reduzieren. Dies gelang im ersten Schritt nicht ganz, konnte aber im Laufe der Zeit immer besser umgesetzt werden, da sich die personelle Situation entspannte. Auch heute gelingt es nicht immer, aber das Signal, das alleine durch den Versuch der Umsetzung gesendet wird, wirkte sich positiv auf die Arbeitszufriedenheit aus.

\section{Vierter Meilenstein für die Personalbindung: Unternehmenskommunikation}

Die Standorte der Diakoniestationen Essen gGmbH sind über das gesamte Stadtgebiet in Essen verteilt. Informationen wurden per E-Mail, in Leitungskonferenzen und Dienstbesprechungen oder über Aushänge weitergegeben. Dennoch war der Informationsverlust sehr hoch. Zur Information aller Mit-

\section{"Der ,Ressource Mitarbeitenders kommt eine wirtschaftliche schlüsselrolle zu«}

sere Mitarbeiter durch die Übertragung zusätzlicher, verantwortungsvoller Arbeitstätigkeiten wie beispielsweise Suchtbeauftragte, Arbeitsschutzbeauftragte, Leitung von Arbeitsgruppen oder Projekten.

Kurzfristig umsetzbar waren Maßnahmen, die Mitarbeitenden über ihre tariflichen und übertariflichen Ansprüche zu informieren. Es wurde ein Mitarbeiterflyer erstellt, der gleichzeitig für die Anwerbung neuer Mitarbeitenden verwendet werden konnte. Den Mitarbeitenden wurden die Flyer in den Dienstbesprechungen vorgestellt und ausgehändigt. Gleichzeitig wurde die Weitergabe an Freunde und Bekannte aus dem Pflegeberuf angeregt. arbeitenden durch die Geschäftsführung über die Entwicklung und strategische Ausrichtung des Unternehmens wurde der Mitarbeiterjahresbrief eingeführt.

Alle übrigen wichtigen Informationen über das Unternehmen werden auf einer durch Passwort geschützten Mitarbeiterseite im Internet veröffentlicht. Gleichzeitig haben aber alle üblichen Kommunikationswege weiterhin Bestand.

\section{Fünfter Meilenstein für die Personalbindung: individuelle Karriereplanung}

Da wir unseren Mitarbeitenden berufliche Entwicklungsmöglichkeiten im Unternehmen bieten wollten, haben 
wir ab Sommer 2012 eine individuelle Karriereplanung für unsere Fachkräfte angeboten. Im ersten Schritt wurden alle Fachkräfte angeschrieben und ihr Interesse an beruflicher Weiterentwicklung abgefragt.

Hieraus kristallisierte sich eine Gruppe, die sich für eine horizontale Personalentwicklung, wie beispielsweise eine Spezialisierung zum Wundmanager oder zur Praxisanleitung, interessierte. Eine andere Gruppe möchte mittelfristig mit Führungsaufgaben betraut werden.

Für die erste Gruppe werden geeignete Fortbildungsmöglichkeiten gesucht und angeboten. Die zweite Gruppe durchlief zunächst ein Assessment, um sich erste Vorstellungen über den Führungsalltag zu machen. Im zweiten Schritt werden nun individuelle Personalentwicklungspläne erarbeitet und es erfolgt nach und nach die Einarbeitung in unterschiedliche Aufgabenfelder auf Leitungsebene.

\section{Erster Meilenstein für die Personalgewinnung: Personalbedarfsplanung}

Grundlage jeglicher Personalgewinnungsstrategie ist eine prospektive Personalplanung. Hierdurch können Entwicklungen im Vorfeld antizipiert und angemessene Maßnahmen entwickelt werden. Eine Reihe von Kennziffern dient dabei der Beobachtung der Entwicklung des Personalbestandes. Zu diesen Kennziffern gehören beispielsweise: Mitarbeiterzahl, Altersstrukturanalyse und Durchschnittsalter, Krankenstand, Bestand Mehrarbeitsstunden, Fluktuation, Anzahl der Bewerbungen und Einstellungen, Anzahl und Zeitpunkt der Kündigungen.

\section{Zweiter Meilenstein für die Personalgewinnung: Entwicklung geeigneter Maßnahmen}

Wie auch bei der Kundengewinnung ist bei der Mitarbeitergewinnung die Empfehlung durch die eigenen Mitarbeitenden einer der besten Wege, neue Fachkräfte zu gewinnen. Die Geschäftsführung und die Gesamtpflegedienstleitung haben dieses Thema gemeinsam mit den Mitarbeitenden im Rahmen der Dienstbesprechungen diskutiert. Zwischenzeitlich können wir sagen, dass unsere Mitarbeitenden die Diakoniestationen Essen immer häufiger als guten Arbeitgeber

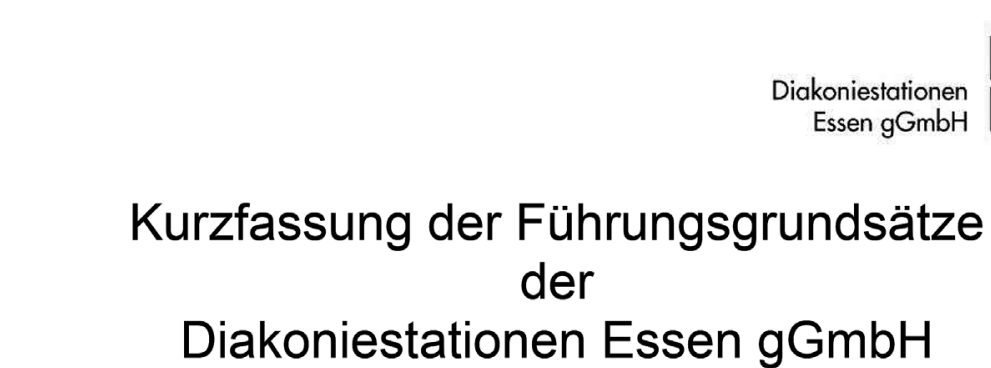

\begin{abstract}
Auf der Grundlage des Leitbildes entwickelten die Führungskräfte in Zusammenarbeit mit den Mitarbeitenden folgenden Werterahmen:
\end{abstract}

- Wir fördern eine Kultur der gegenseitigen Wertschätzung und Eigenverantwortlichkeit.

- Wir stellen einen für die Arbeit zeitnahen Informationsaustausch sicher und treffen verbindliche Absprachen.

- Anregungen zur Weiterentwicklung und konstruktiver Kritik geben wir Raum.

- Offene und direkte Kommunikation sind für uns der Weg zu Transparenz und Vertrauen.

- Wir stärken die Eigenverantwortung sowie die Eigeninitiative jedes Einzelnen und fördern die Zusammenarbeit.

- Wir verantworten klare und erreichbare Ziele, die wir im Team zu erreichen suchen.

- Eine professionelle Grundeinstellung führt zu Freude und Überzeugung an und in unserer Arbeit.

\title{
14. März 2012
}

Martina Pollert Geschäftsführerin

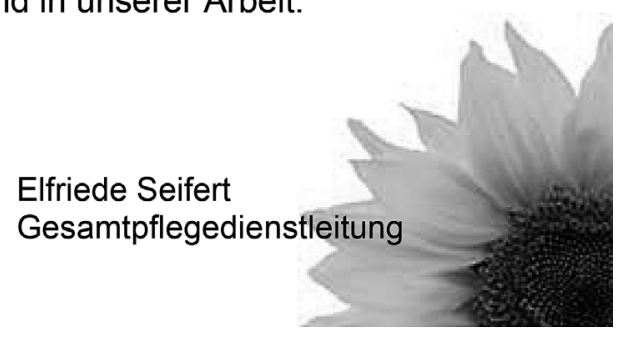

Ein fairer Umgang der Leitungskräfte mit ihren Mitarbeitenden ist ein bedeutsamer Faktor bei der Arbeitszufriedenheit. Leitsätze können einen anerkennenden Führungsstil fördern.

weiterempfehlen. Weitere erfolgreiche Instrumente der Personalgewinnung waren: frühzeitige Investition in Ausbildung, Nutzung von Online-Medien, familienfreundliche Arbeitszeitmodelle, frühzeitige Investition in Weiterbildung.

\section{Resümee: Die Erfolge}

Alle getroffenen Maßnahmen spiegeln in Ihrer Gesamtheit eine für uns wichtige Unternehmensgrundhaltung im Unternehmen wider. Der zahlenmäßige Erfolg bestätigt, dass wir uns auf dem richtigen Weg befinden (auch wenn wir sicher noch nicht am Ziel angekommen sind):
- Im Jahr 2012 lagen uns 271 Bewerbungen vor, von denen wir 53 eingestellt haben.

- Die Mehrarbeitsstunden haben sich bis Ende 2012 fast halbiert.

- Die Kosten für Zeitarbeit liegen seit dem vierten Quartal 2012 bei Null Euro.

Wir haben es uns zur Aufgabe gemacht, ein attraktiver Arbeitgeber zu sein, der seinen Beschäftigten gute Arbeitsbedingungen bietet und jeder Mitarbeiterin und jeden Mitarbeiter unabhängig von der Position im Unternehmen respektvoll behandelt. 